\title{
Cryptophagidae (Coleoptera) of the KwaZulu Natal (South Africa)
}

\section{Cryptophagidae (Coleoptera) провинщии Квазу́лу-Ната́ı (ЮАР)}

\author{
G.Yu. Lyubarsky \\ Г.Ю. Аюбарский
}

Zoological Museum of Moscow State University, Bol'shaya Nikitskaya str. 6, Moscow 125009, Russia. Зоологический музей МГУ, ул. Б. Никитская 6, Москва 125009, Россия.

KEY WORDS: Coleoptera, Cryptophagidae, KwaZulu-Natal, South Africa Republic.

КЛЮЧЕВЫЕ СЛОВА: Coleoptera, Cryptophagidae, Квазу́лу-Ната́л, ЮАР.

ABSTRACT. The material KwaZulu-Natal's Collection: the results of expedition (2003-04) in South Africa Republic. The some species Cryptophagus, Micrambe, Atomaria, Curelius are determined.

РЕЗЮМЕ. Представлен материал, собранный во время экспедиции в ЮАР в 2003-04 гг. Найдены виды родов Cryptophagus, Micrambe, Atomaria, Curelius.

The family Cryptophagidae comprises about 800 species worldwide. Cryptophagidae [in the sense of Johnson et al., 2007] are to be found in mouldy plant substrates, in flowers, in fungi, under bark, and in the nests of social insects, and the nests of birds and mammals. The species associated with stored food products are supposed to live on the spores and hyphae of moulds [Hinton, 1945].

The material was collected by M.B. Mostovski in KwaZulu-Natal province (Fig. 1). The KwaZulu-Natal National Park specialises in the conservation of plants

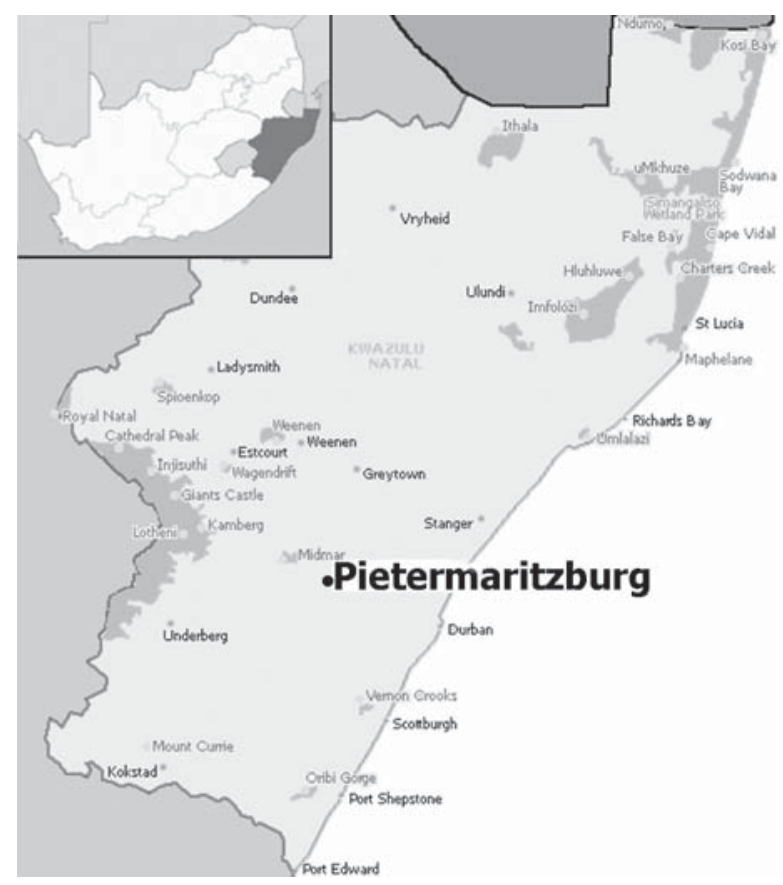

Fig. 1. Prov. KwaZulu-Natal in South Africa Republic. Рис. 1. Карта провинции Квазу́лу-Ната́л (ЮАР). and animals from the eastern region of South Africa and of rare and endangered species from elsewhere. A total of thirty-seven species and subspecies endemic to the Province in certain families of Coleoptera, Diptera, Lepidoptera, Mecoptera and Odonata were included [Armstrong, 2002]. This material was received from V.G. Gratchev, recently deceased entomologist dealing with family Curculionidae.

Besides Cryptophagidae, material contains several genera of Phalacridae. Phalacridae of South Africa is poorly understood, except old papers of Champion and Guillebeau [Champion, 1925; Guillebeau, 1892, 1893, 1894] and several contemporary works [Lyubarsky, 1997, 2004; Svec, 2002]. The material contains Entomocnemus Guillebeau, 1894, Augasmus Motschulsky, 1858, Olibrus Erichson, 1845, Phalacrus Paykull, 1800, Tinodemus Guillebeau, 1894.

The present work contains also determination of Cryptophagid species. Material available for study is presented by 429 specimens of Cryptophagidae, all collected by M.B. Mostovski (2003-04). The material derives mostly from Pietermaritzburg. Standard label: S. Africa, KwaZulu-Natal, Pietermaritzburg, Hilton, Malaise trap, leg. M.B. Mostovski.

\section{Family Cryptophagidae Kirby, 1837 \\ Subfamily Cryptophaginae Kirby, 1837 \\ Cryptophagus laticollis Lucas, 1846}

MATERIAL. S. Africa, KwaZulu-Natal, Pietermaritzburg, Hilton, Malaise trap, 23.109-12.11.2003 leg. M. Mostovski (2 spec.); KZN, PMB, Hilton, Malaise-tr./ garden, 27.09-13.10.2003 leg. M. Mostovski (3 spec.)

DISTRIBUTION. A cosmopolitan distribution, synanthrop.

\section{Micrambe sp.indet.}

MATERIAL. S. Africa, KwaZulu-Natal, Pietermaritzburg, Hilton, Malaise trap, 23.109-12.11.2003, leg. M. Mostovski (49 spec.); ibid. 13-23.11.2003, leg. M. Mostovski (25 spec.); ibid. 24.1109.12.2003, leg. M. Mostovski (52 spec.); ibid. 109-23.12.2003, leg. M. Mostovski (24 spec.); ibid. 24.12.2003-14.01.2004, leg. M. Mostovski (31 spec.); ibid. 15-26.01.2004, leg. M. Mostovski (6 spec.); ibid. 27.01-16.02.2004, leg. M. Mostovski (47 spec.); ibid. 17-29.02.2004, leg. M. Mostovski (18 spec.); ibid. 29.0213.03.2004, leg. M. Mostovski (34 spec.); ibid. 3-5.03.2004, leg. M. Mostovski (3 spec.); S. Afr., KZN, PMB, Hilton, Malaise-tr./ garden, 27.09-13.10.2003, leg. M. Mostovski (79 spec.). 


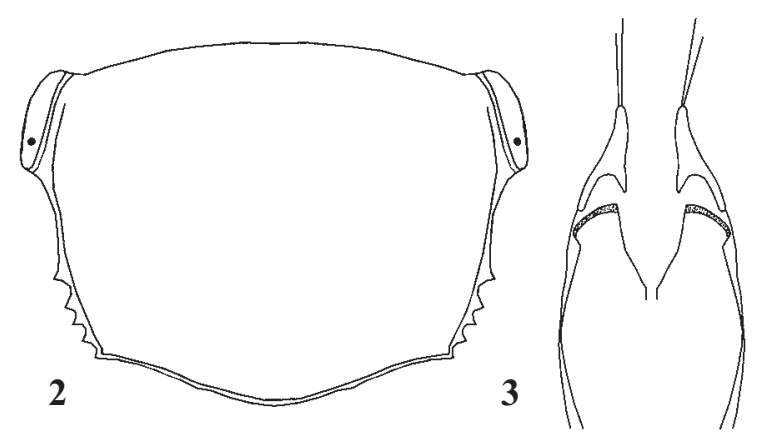

Figs 2-3. Micrambe sp.indet.: 2 - pronotum, 3 - aedeagus. Рис. 2-3. Micrambe sp.indet.: 2 - переднеспинка; 3 - эдеагус.

REMARKS. This species (Figs 2-3) is distinguished by the following features: pronotum very transverse, four teeth in lateral margin, pubescence elated, hind wing present.

$\mathrm{N}$. Bruce in several major articles describing a new species in the genus Micrambe from South Africa. Three authors (Bruce, Grouvelle, and Scott) describe several dozen species of the genus from South Africa. This genus requires a detailed revision. For example, some species are described by a single female. These multiple forms have been described by Bruce [1951, 1952a, b, 1954, 1959, 1960, 1963, 1965], Grouvelle [1903, 1908, 1916, 1919], Scott [1935, 1934-1936].

Listed criteria (pronotum very transverse, four teeth in lateral margin, pubescence elated, hind wing present) have not all species. The some species are not suitable for the specified in the description of special features. For instead, M. varicolor Grouvelle, 1919 - last sternite of abdomen with depression, metanotum with narrow not punctured strip medially.

Many species are suitable on the basis of descriptions: $M$. brachyptoracicus Bruce, 1952 (Tanganyika ); M. hirsutus Grouvelle, 1916 (Cape); M. irritabilis Bruce, 1951 (Park Albert, Congo); M. kolbei Grouvelle, 1916 (Zululand); $M$ madagascariensis Grouvelle, 1916 (Mt. Cameroon); M. minutus Grouvelle, 1916 (Cape); M. molestus Bruce, 1951 (Park Albert, Congo); M. mutilatus Bruce, 1952a (Congo); M. olkokolae Bruce, 1960 (Tanganyika); M. plagiatus Pering. (Cape); M. punctatus Grouvelle, 1916 (Natal); M. reitteri Grouvelle, 1916 (Cape); M. simoni Grouvelle, 1916 (Natal); $M$. tenuicornis Grouvelle, 1908 (Rodesia).

\section{Subfamily Atomariinae LeConte, 1861}

Atomaria (Anchicera) lewisi Reitter, 1877

MATERIAL. S. Africa, KwaZulu-Natal, Pietermaritzburg, Hilton, Malaise trap, 23.10-12.11.2003, leg. M. Mostovski (6 spec.); ibid. 10-23.12.2003, leg. M. Mostovski; ibid. 24.12.200314.01.2004, leg. M. Mostovski (4 spec.); ibid. 17-29.02.2004, leg. M. Mostovski; S. Afr., KZN, PMB, Hilton, Malaise-tr./ garden, 27.09-13.10.2003, leg. M. Mostovski (15 spec.).

DISTRIBUTION. A cosmopolitan distribution, synanthrop. There are findings in South Africa: Cape Prov. [Johnson, 1970].

Atomaria (Anchicera) marshalli Johnson, 1968

MATERIAL. S. Afr., KZN, PMB, Hilton, Malaise-tr./ garden, 27.09-13.10.2003, leg. M. Mostovski (4 spec.).

DISTRIBUTION. Endemic to South Africa. There are findings in South Africa: Estcourt, Cape Prov. [Johnson, 1968, 1970].

\section{Curelius japonicus (Reitter, 1877)}

MATERIAL. S. Africa, KwaZulu-Natal, Pietermaritzburg, Hilton, Malaise trap, 13-23.11.2003, leg. M. Mostovski; ibid. 24.12.2003-14.01.2004, leg. M. Mostovski (6 spec.); ibid. 1526.01.2004, leg. M. Mostovski (4 spec.); ibid. 27.01-16.02.2004, leg. M. Mostovski; ibid. 17-29.02.2004, leg. M. Mostovski (9 spec.); ibid. 29.02-13.03.2004, leg. M. Mostovski (4 spec.).
DISTRIBUTION. Common in W. Africa: Senegal, Gambia, Sierra Leone, Ghana, Nigeria. Are distributed on the eastern coast of Africa from Ethiopia to Kenya, south Zimbabwe, as well as in USA and Brazil [Johnson, 1989].

\section{References}

Armstrong A. J. 2002. Insects and the determination of priority areas for biodiversity conservation in KwaZulu-Natal province, South Africa // African Entomology. Vol.10. No.1. P.11-27.

Bruce N. 1938. Revision critique des especes du genre Cryptophagus determines par A. Grouvelle// Rev. franc. d'Ent. Vol.5. P.246.

Bruce N. 1951. Cryptophagidae // Exploration du Parc National Albert . Bruxelles. 26 pp.

Bruce N. 1952a. Coleoptera Cryptophagidae aus dem Congo-Gebiet im Musee Royal du Congo belge// Rev. Zool. Bot. Afr. Vol.46. No.3-4. P.386-395.

Bruce N. 1952b. Coleoptera Cruyptophagidae in the British Museum I // Ann. Mag. Nat. Hist. Ser.12. No.5. P.167-188.

Bruce N. 1954. Einige Coleoptera (Cryptophagidae) aus Ost- und Westindien und Sudafrika im Zoologischen Museum, Amsterdam // Entomologische Berichten. Bd.15. S.172-175.

Bruce N. 1959. Cryptophagidae (Coleoptera, Cucujoidea) // Exploration du Parc National de la Garamba. Vol.17. P.3-13.

Bruce N. 1960. LXIII. Coleoptera Cryptophagidae// Annales Musée royal de l'Afrique centrale. Sci. zool. Vol.148. P.2509-266.

Bruce N. 1963. Coleoptera Cryptophagidae in Musee Royal de l'Afrique Centrale (V) // Rev. Zool. Bot. Afr. T.67. No.3-4. P.203-221.

Bruce N. 1965. Coleoptera Cryptophagidae in Musee Royal de l'A Arique Centrale (VI) // Rev. Zool. Bot. Afr. T.71. No.1-2. P.34-48.

Champion C.G. 1925. Studies in Phalacridae (I.) - S. and E. African forms (Coleoptera) // Annals and Magazine of Natural History. Vol.15. P.35-53.

Grouvelle A. 1903. Voyage de M. Maurice Mandron dans l'Inde Meridionalee (Coleoptera) // Ann. Soc. Ent. Fr. Vol.72. P.343.

Grouvelle A. 1908. Coleopteres recoltes dans l'Afrique Orientale allemande // Revue d'Entomologie. Vol.27. P.191.

Grouvelle A. 1916. Descriptions d'especes nouvelles de Cryptophagidae // Memoires Ent. Paris.Vol.30.

Grouvelle A. 1919. Descriptions de Genres nouveaux et d'especes nouvelles // Memoires Ent. Vol.70.

Guillebeau F. 1892. Revision des Phalacrides de la faune Palearctique // Revue d'Entomologie. T.2. P.141-197.

Guillebeau F. 1893. Description de deux especes de Phalacrides recueillies d'Aden par M. E. Simon // Annales de la Societe Entomologique de France. T.62. P.297-298.

Guillebeau F. 1894. Descriptions de quelques especes de la famille des Phalacridae de la Collection de M.Antoine Gouvelle // Annales de la Societe Entomologique de France. T. 63. P.275-310.

Hinton H.E. 1945. A monograph of the beetles associated with stored products. Vol.I. London: Brit. Mus. Nat. Hist. 433 pp.

Johnson C. 1968. Some interesting species of Atomaria Stephens (Col., Cryptophagidae) in the British Museum // Entomologist. Vol.101. P.228-231.

Johnson C. 1970. Coleoptera: Creyptophagidae: Atomariinae. // South African animal life. Results of the Lund University Expedition in 1950-1951. Vol.14. P. 228-229.

Johnson C. 1989. Cryptophagidae (Coleoptera) of the Arabian Peninsula // Fauna of Saudi Arabia. Vol.10. P.108-112.

Johnson C., Otero J.C. \& Leshen R.A.B. 2007. Cryptophagidae // I. Löbl, A. Smetana (eds.). Calalogue of Palaearctic Coleoptera. Stenstrup: Apollo Books. Vol.4. P.513-531

Lyubarsky G. Yu. 1997. Phalacridae of the Southern Africa (Coleoptera) // Russian Entomological Journal. Vol.6. No.1-2. P.17-40.

Lyubarsky G.Y. 2004. New species and new data on Phalacridae (Coleoptera) of the Republic of South Africa // Russian Entomological Journal. Vol.13. No.3. P.123-125.

Scott H. 1935. Coleoptera from east Africa // J. Linn. Soc. Lond. Zool. Vol.39. P.252-281.

Scott H. 1934-1936. Coleoptera associated with the giant Lobelias and arborescent Senecious of Eastern Africa (Cryptophagidae) // J. Linn. Soc. (Zool.). Vol.39. P.252-279.

Svec Z. 2002. Revision of the African species of the genus Tinodemus Guillebeau (Coleoptera, Phalacridae) // Mitteilungen aus dem Zoologischen Museum zu Berlin. Vol.78. No.2. P.217-256. 